\title{
La Interculturalidad en Extremadura: tareas pendientes para una escuela inclusiva del s. XXI
}

\section{Interculturality in Extremadura: Pending Tasks for a 21 st Century Inclusive School}

\author{
Raquel Borrero López* \\ raquel.borrer@salesianosbadajoz.com \\ Florentino Blázquez Entonado ${ }^{1 * *}$ \\ blazento@unex.es \\ *Colegio Ramón Izquierdo, Badajoz, España \\ **Universidad de Extremadura, España
}

\begin{abstract}
Resumen:
La Comunidad Autónoma de Extremadura es una de las últimas regiones del mundo occidental que ha incorporado a sus escuelas un notable número de alumnos de origen extranjero o de minorías étnicas y culturales. Esta investigación pretende comprobar el modelo pedagógico desde el que se entiende y trabaja la educación intercultural por parte del profesorado, detectar sus necesidades formativas y sugerir las claves que posibiliten el desarrollo del enfoque crítico intercultural en los centros educativos de Infantil, Primaria y Secundaria de la región.

Para responder a tales objetivos se ha realizado un diseño mixto de investigación utilizando cuestionario, entrevistas, grupos focales de discusión y análisis documental, cuyo conjunto pretende fortalecer la validez de la investigación. Las numerosas conclusiones requieren muchos matices, pero se aprecia una escasa repercusión en la práctica de la percepción que tiene
\end{abstract}

\begin{abstract}
:
The Autonomous Community of Extremadura (Spain) is one of the last regions in the Western world to incorporate to its schools a remarkable number of students of foreign origin or ethnic and cultural minorities. This research aims to verify the pedagogical model from which intercultural education is understood and worked by teachers with a view to detecting their training needs and to suggest the keys that allow for the development of the intercultural critical approach in primary and secondary schools in the region. To respond to these objectives, a mixed research design was carried out using questionnaires, interviews, discussion focus groups and documentary analysis. The conclusions obtained are rather nuanced but there is a slight impact on the teachers' perception of multiculturalism, especially regarding their daily work in the classroom and the communication and disciplinary models that are used in it. However, even when
\end{abstract}

1 Dirección para correspondencia (correspondence address):

Florentino Blázquez Entonado. Dpto. de Ciencias de la Educación. Universidad de Extremadura. Facultad de Educación. Campus universitario. Avda. de Elvas, s/n. 06006 Badajoz (España). 
La Interculturalidad en Extremadura: tareas pendientes para una escuela inclusiva del s. XXI

Raquel Borrero López y Florentino BlázQuez Entonado

el profesorado sobre la multiculturalidad, especialmente en el trabajo cotidiano en el aula y los modelos de comunicación y disciplinares que en ella se emplean. Ahora bien, aun cuando el profesorado se encuentra todavía lejos de haber interiorizado un modelo educativo intercultural crítico, se aprecian preocupación e interés por los grupos minoritarios, lo que constituye un firme punto de partida para el trabajo formativo.

\section{Palabras clave:}

Educación Intercultural; Multiculturalismo e Interculturalidad; Educación de minorías étnicas. the teachers are still far from internalizing a critical intercultural educational model, they express concern and interest regarding minority groups, which constitutes a strong starting point for training work.

\section{Keywords:}

Intercultural Education; Multiculturalism and Interculturality; Education of ethnic minorities.

\section{Résumé:}

La Communauté Autonome d'Estrémadure est l'une des dernières régions du monde occidental qui a intégré dans ses écoles un nombre remarquable d'étudiants d'origine étrangère ou issus de minorités ethniques et culturelles. Cette recherche a pour but de vérifier le modèle pédagogique depuis lequel l'éducation interculturelle est comprise et travaillée par les enseignants, d'identifier leurs besoins de formation et de suggérer les clés qui permettent le développement d'une approche critique interculturelle dans les centres d'éducation maternelles, primaires et secondaires de la région. Afin de répondre à ces objectifs, une recherche de type mixte a été réalisée à l'aide de questionnaires, d'entretiens, de groupes de discussion et d'analyse documentaire, dont l'objectif est de renforcer la validité de la recherche. Les nombreuses conclusions nécessitent de plusieurs nuances, mais il y a peu d'impact sur la pratique de la perception qu'ont les enseignants du multiculturalisme, en particulier dans le travail quotidien en classe et dans les modèles de communication et de discipline qui y sont utilisés. Cependant, même si les enseignants sont loin d'avoir intériorisé un modèle éducatif interculturel critique, on remarque une certaine préoccupation et un intérêt pour les groupes minoritaires, ce qui constitue un point de départ solide pour la formation.

\section{Mots clés:}

Education interculturelle; multiculturalisme et interculturalité; Education des minorités ethniques.

Fecha de recepción: 10-2-2018

Fecha de aceptación: 14-5-2018

\section{Introducción}

La sociedad del siglo XXI se pregunta si puede encontrar en la escuela el camino para construir una identidad colectiva, aunque en los denominados tiempos "posmodernos" adolezcamos de los apoyos hasta hace muy poco reconocidos como universales. El hecho es que vivimos en una era 
caracterizada por muchas incertidumbres y turbulencias sociopolíticas. La oportunidad, el acceso y los bienes materiales se distribuyen de manera claramente injusta, lo que resulta ser, aunque rara vez se entiende, una forma de violencia particularmente nociva en un mundo en el que los recursos existen para proporcionar bienestar a todos. Una filosofía por la que el profesor Escudero Muñoz, a quien se dedica este artículo, ha trabajado toda su vida académica en defensa de una escuela en la que puedan convivir y prepararse para la plena ciudadanía grupos tradicionalmente marginados. Con este modesto trabajo sobre su querida tierra de origen y sobre una temática tan próxima a su honda conciencia social, nos unimos al merecido homenaje académico, reforzando, por otro lado, una histórica e indeleble amistad.

Unidos a sus inquebrantables objetivos, alentamos a los educadores a lidiar con la injusticia global contemporánea, propia también de la tierra extremeña que compartimos, como una llamada a los proponentes de la educación inclusiva para reimaginar la educación a la luz de las realidades sociopolíticas y económicas actuales, en consideración a formas de opresión nuevas, cambiantes y que, desafortunadamente, parecen caminar hacia peor. Esto a pesar de que desde los años setenta constatemos una sólida trayectoria de estudios tanto europeos como americanos que contemplaban como objetivo compartido fomentar la dimensión intercultural de la educación, preparando al alumnado para vivir en una sociedad caracterizada por la diversidad lingüística y cultural.

Tal constatación no descarta, más bien al contrario, que la educación intercultural puede tener la respuesta a cómo educar desde la diversidad para la promoción de sociedades cohesionadas con sentido de comunidad, donde se puedan desarrollar la cooperación, la solidaridad y la inclusión. Y es indudable que la escuela puede y debe asumir esta nueva demanda: educar a ciudadanos y ciudadanas que ayuden a hacer posible una sociedad intercultural (Gundara, 2010: 231).

\section{Extremadura y su realidad multicultural}

España deja de ser a finales de los años 70 un país de emigrantes para convertirse, entre mediados de los 90 y principios del siglo XXI en uno de los países con mayores tasas de acogida de inmigrantes (superando con creces a países como Estados Unidos o Francia), todo ello promovi- 
La Interculturalidad en Extremadura: tareas pendientes para una escuela inclusiva del s. XXI

Raquel Borrero López y Florentino BlázQuez Entonado

do por diferentes circunstancias de estabilidad política y económica, así como por condiciones geográficas. Y por eso en los últimos años han ido proliferando las producciones y difusión de investigaciones, publicaciones y actividades formativas por parte de grupos de investigación centrados en este campo de estudio y de gran repercusión, como el Proyecto Integrado Includ-ed, la Red_Inter, etc.

Extremadura es una de las últimas regiones del mundo occidental a las que han Ilegado las migraciones en masa que han propiciado trabajos de investigación y divulgación científica, prioritariamente en los ámbitos de la Política, el Derecho, la Sociología, la Educación y la Antropología por todo el mundo.

Ni multiculturalidad ni interculturalidad pueden considerarse fenómenos novedosos o desconocidos, aunque en su calidad de conceptos dinámicos, han tenido que adaptarse a las peculiaridades de los últimos años y a las repercusiones que los nuevos asentamientos de migrantes han producido en las sociedades de acogida. Consideramos en este trabajo la cohesión social como un buen rasgo diferencial entre multiculturalidad e interculturalidad, ya que este concepto implica más que una mera coexistencia de culturas diferentes, que podría desprenderse de la idea de multiculturalidad, implicando el desarrollo de unos valores comunes y una cultura cívica, un cierto orden y control social, la existencia de solidaridad social y la reducción de las disparidades en la riqueza (Ponce, 2007: 51-52), esto es, un tipo de respuesta generada en un espacio y un tiempo concretos. Es por este motivo por el que resulta tan importante conocer las realidades y ajustar los modelos y las teorías existentes a las necesidades concretas. Sólo de este modo podremos evitar generalizar experiencias sin criterio o repetir modelos erráticos reformulando propuestas aplicadas a otras regiones.

En este orden de cosas consideramos la tardía llegada de este fenómeno a nuestras escuelas como un regalo envenenado por dos motivos principales: por la falta de preparación para desarrollarnos en una nueva sociedad en ámbitos cruciales (política, sensibilización social, economía y mercado de trabajo, formación del profesorado y del alumnado, etc.) a pesar de haber contado con más tiempo que otras comunidades autónomas para ello; y por el enorme riesgo de reducir el trabajo a la mera repetición de patrones y modelos aplicados previamente (en otros contextos, ante otras realidades) sin un previo, profundo y crítico análisis de nuestra propia situación, partiendo del convencimiento de que el 
centro escolar no ha de ser un receptor pasivo del mundo, sino el laboratorio desde el que se inventen respuestas capaces de generar cambios.

La escuela extremeña intenta ofrecer respuestas transformadoras de la dinámica realidad social. Con estos fines se vienen celebrando múltiples actividades como Congresos, Simposios, Reuniones científicas y una notable producción de grupos universitarios de investigación. Sin embargo y a pesar de la proliferación de actividades, no hemos encontrado publicado ningún estudio actual que trate de describir la realidad intercultural extremeña desde la perspectiva de los profesionales (docentes, asesores, orientadores, etc.) que realice propuestas para el desarrollo docente en el marco de una comprensión inclusiva de la interculturalidad: por las dimensiones que ha cobrado el fenómeno de acogida de población inmigrante en le región, por el importante cota de tasas de escolarización de alumnado de origen extranjero en nuestros centros educativos y por la existencia de población escolar de "segunda generación" en varias zonas de la región.

En la Comunidad Autónoma de Extremadura la multiculturalidad, como característica propia de las sociedades modernas en los países desarrollados, se ha hecho presente de manera diferente en sus dos provincias. Para esta descripción general de la diversidad cultural contemplada en Extremadura y salvo la consulta a dos obras existentes (Castro, 2007 y León, Gómez, Felipe, Gonzalo y Latas, 2008) se han sintetizado análisis de datos estadísticos oficiales de los años durante los cuales se ha venido realizando la investigación partiendo de fuentes como los Planes Estratégicos de Ciudadanía e Inmigración, el INE, la Junta de Extremadura, etc. Antes de que en 2006 se produjera el boom de las migraciones, procedentes principalmente de África, Latinoamérica, Europa del Este y Asia, la Junta de Extremadura había lanzado medidas de intervención para trabajar con el colectivo de alumnado extranjero escolarizado en determinadas zonas de Cáceres. La procedencia geográfica y los motivos de la llegada de la población inmigrante han sido dos grandes condicionantes de las clarísimas diferencias que los datos reflejan sobre el mapa social de Cáceres y Badajoz. En aquel momento, Badajoz no podía considerarse profundamente tocada por esta realidad. Sin embargo, en tan sólo un año, la situación comenzó a cambiar tan rápidamente que ni administraciones, ni centros educativos pudieron responder a las demandas con la suficiente antelación y preparación. Ha sido ésta una de las principales características del proceso de cambio en la provincia de Badajoz: la rapidez con la que la 
La Interculturalidad en Extremadura: tareas pendientes para una escuela inclusiva del s. XXI

Raquel Borrero López y Florentino BlázQuez Entonado

realidad multicultural se ha hecho presente en las escuelas y la necesaria adaptación in situ que administraciones y profesionales del ámbito de la educación se han visto obligados a realizar.

Extremadura, como el resto de comunidades españolas, ha experimentado un detrimento de la población inmigrante procedente de la Europa Comunitaria, a favor de un aumento progresivo del resto de procedencias que serían, en orden de importancia: África (Magreb y zona subsahariana principalmente), Latinoamérica, Rumanía y Asia. Aunque sigue habiendo población temporera, la mayoría de la población inmigrante se ha establecido, incluso con sus familias, gracias a las medidas de reagrupación familiar. Continuando con los datos generales, la mayoría de la población son hombres, principalmente entre los extranjeros procedentes de África y Asia, mientras que únicamente en el caso de América, la población es mayoritariamente de mujeres. Se trata de una población joven, con casi un $90 \%$ menores de 50 años. La principal ocupación son las campañas agrícolas, seguidas de ocupaciones en hostelería, construcción y servicio doméstico. La concentración suele ser rural, aunque en el caso de los extranjeros procedentes de Asia, la práctica totalidad reside en los municipios, concentrándose la mayoría en la provincia de Badajoz. La amplia movilidad de este colectivo atendiendo a la oferta de empleo y la saturación, en ocasiones, de los servicios sociales, sanitarios y educativos de las zonas en las que residen, son las dos principales circunstancias que caracterizan las dificultades de inclusión social que encuentran.

De forma específica, Badajoz cuenta con el $61 \%$ de la población extranjera, mientras que el 39\% restante reside en la provincia de Cáceres. El municipio con mayor tasa de extranjeros de Badajoz es Almendralejo, mientras que en Cáceres es Talayuela.

La mayoría de la población inmigrante en Badajoz procede de Rumanía y de Latinoamérica; en Cáceres de Marruecos e igualmente de Latinoamérica. Los asentamientos principales se localizan en la Zona de Barros, en Badajoz y en las zonas de Campo Arañuelo y La Vera, en Cáceres. En resumen, entre el año 2000 y la fecha actual, el panorama extremeño ha girado 90 grados. Mientras que Cáceres fue durante muchos años la provincia con mayor número de inmigrantes, con prácticamente el 70\%, esta posición la ocupa ahora Badajoz, casi en la misma proporción. Así, no es de extrañar que en la mitad de este trayecto, en torno al año 2005, cuando ya se hablaba en Talayuela de aulas de inmersión 
lingüística y de mediación intercultural, en Badajoz las únicas prácticas educativas interculturales giraban en torno al alumnado gitano.

Atendiendo ya al alumnado de origen extranjero escolarizado, el número gira en torno a los 5500, de los cuales, menos del 50\% procede de países de habla hispana. La mayoría del alumnado se escolariza en Primaria, seguida de Infantil y, por último, de ESO, Bachillerato y Ciclos Formativos. El 90\% se escolariza en centros públicos. Una característica importante en torno a la escolarización del alumnado inmigrante en Extremadura es la irregularidad o asistencia discontinua a clase por frecuentes traslados a otras zonas de España o por incompatibilidad de calendario escolar con las celebraciones religiosas y festividades de sus respectivas culturas.

Como venimos señalando, las características de las dos provincias extremeñas difieren y el hecho multicultural ha evolucionado en ambas a diferentes ritmos. Determinadas zonas de la provincia de Cáceres cuentan con modelos de intervención estudiados y consolidados que, efectivamente, marchan muy por delante de las respuestas ofrecidas desde la provincia de Badajoz, motivo por el que hemos considerado importante realizar este estudio, con el objeto de tratar de promover un modelo educativo común.

Por la imposibilidad de reflejar toda la normativa regional, que es relativamente amplia y diversa, aunque probablemente insuficiente y no del todo generalizada, reflejamos resumidos en el siguiente cuadro los programas educativos que Extremadura trabaja específicamente en determinados centros de la región.

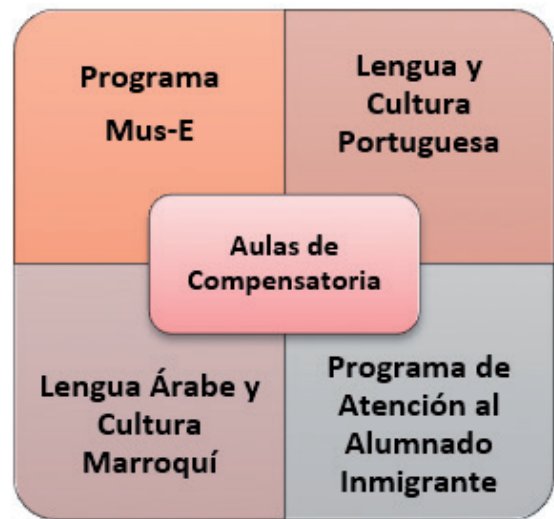

Figura 1. Programas Educativos Multiculturales de Extremadura. Elaboración propia. 
La Interculturalidad en Extremadura: tareas pendientes para una escuela inclusiva del s. XXI

Raquel Borrero López y Florentino BlázQuez Entonado

\section{Interculturalidad y profesorado}

Para nosotros la interculturalidad reconoce la naturaleza intersujetos del conocimiento y de las acciones y expresa un proceso de aprendizaje y de enriquecimiento mutuo de las culturas en contacto. De acuerdo con Arnáiz y de Haro (2004), nosotros también partimos de un concepto de interculturalidad que entiende la diversidad como el núcleo de todo el proceso educativo.

Dicho esto, frente al reto de la multiculturalidad y desde el ámbito educativo, el enfoque desde el que se plantea este estudio y desde cuya perspectiva pretendemos entender la educación intercultural no es otro que el Paradigma Holístico Multicultural propuesto hace años por Banks y Lynch (1986). De esta manera entendida, la escuela sería un todo interrelacionado, un medio integrado por una serie de variables identificables que reflejan igualdad social, cultural y étnica. El contexto escolar debería ser un medio cultural donde la aculturación tuviera lugar. Profesorado y alumnado deberían asimilar puntos de vista, percepciones e identidad del otro al interactuar entre sí, de modo que unos y otros se vieran enriquecidos en el proceso, mejorando la situación académica de los alumnos de las diversas culturas al verse reflejados y legitimados en la escuela (Aguado, 2003).

Este paradigma supone la necesidad de que los procesos de aculturación y acomodación se den simultáneamente en el medio escolar. Cuando hay acomodación, los grupos con diversas culturas pueden mantener su identidad conviviendo en pacífica interacción. La escuela debe ayudar a los estudiantes a desarrollar nuevas identificaciones, aptitudes y actitudes necesarias para funcionar efectivamente en la cultura comunitaria. Por eso, entendemos por centros educativos interculturales aquellas instituciones educativas preocupadas y ocupadas en emplear pedagógicamente la diversidad cultural como oportunidad y riqueza. La educación intercultural ofrece la oportunidad de entender la escuela no solo como espacio de formación, sino como lugar de encuentro de culturas para una educación democrática e integral, capaz de fortalecer la convivencia escolar.

El profesor Esteve (2004) entendía la educación intercultural como un desafío pedagógico, generador de nuevos modelos formativos que aún no han terminado de desarrollarse. En este sentido creemos que la diversidad social, cultural y lingüística se constituyen en un motor de 
cambio y empuje de una escuela democrática e inclusiva, un mecanismo en definitiva, de renovación pedagógica. La educación intercultural se transforma en una propuesta de acción educativa de carácter teóricopráctico promotora del intercambio de valores solidarios y democráticos. Nos encontramos, por tanto, ante un reto pedagógico y organizativo que está marcando la actualidad educativa y la evolución de la educación de nuestro país. Por eso, como proponen Palaiologou y Gorski (2017) es importante crear nuevos espacios políticos y académicos que permitan la construcción de valores educativos y públicos compartidos.

Desde esta perspectiva, abogamos por tratar de contrarrestar estas percepciones mediante propuestas que aborden la educación intercultural desde una orientación crítica y comprometida con la sociedad; desde modelos de formación que busquen la modificación de comportamientos, actitudes y percepciones (Blázquez y Borrero, 2012) y desde una perspectiva que no entienda la educación intercultural como un "problema añadido" a la ya etiquetada como problemática atención a la diversidad.

Nuestra intención es repensar los modelos, procesos y estrategias con los que trabaja actualmente el profesorado de los centros educativos, atendiendo a los principios de diversidad curricular y de inclusión educativa. A pesar de contar con múltiples y serias propuestas, las instituciones formadoras de profesorado aún no han adoptado el compromiso serio de trabajar con el profesorado en el desarrollo de todas las dimensiones que constituyen la competencia intercultural como profesionales de la enseñanza.

En este sentido, este trabajo pretende identificar percepciones y opiniones del profesorado acerca la intervención educativa con alumnado de origen extranjero o de minorías étnicas y culturales y comprobar el modelo pedagógico desde el que el profesorado entiende y trabaja la educación intercultural en los centros educativos extremeños. Con ello delimitaríamos el modelo que administraciones y centros educativos están empleando en el desarrollo de la educación intercultural en Extremadura.

Así mismo, se pretenden detectar las necesidades formativas del profesorado desde la perspectiva de un modelo pedagógico centrado en la interculturalidad que ayuden a determinar las claves de una formación del mismo basada en el desarrollo de la competencia intercultural. 
La Interculturalidad en Extremadura: tareas pendientes para una escuela inclusiva del s. XXI

Raquel Borrero López y Florentino BlázQuez Entonado

\section{Diseño de la investigación}

Para responder a la amplitud de los objetivos se ha decidido un diseño mixto de investigación por encuesta y a través de entrevistas y grupos focales de discusión, más un análisis documental, cuyo conjunto pretende fortalecer la validez de la investigación.

La investigación se inicia en 2009 y queda conformada por tres estudios: uno primero sobre la Educación Intercultural en Badajoz que analiza los Programas de Atención al Alumnado Inmigrante de la provincia; un segundo sobre la Educación Intercultural en Extremadura ampliando la muestra e incluyendo tanto a Badajoz como a Cáceres, en el que se realiza un análisis pedagógico desde la perspectiva docente; y un tercero, que complementa y enriquece de manera transversal a los anteriores con el análisis de proyectos interculturales de centros educativos extremeños, a partir de la documentación institucional de una amplia muestra de centros. Este artículo se limita a un periodo de investigación de 5 años comprendidos entre 2012 y 2016, donde se presentamos los resultados de los dos últimos estudios referidos.

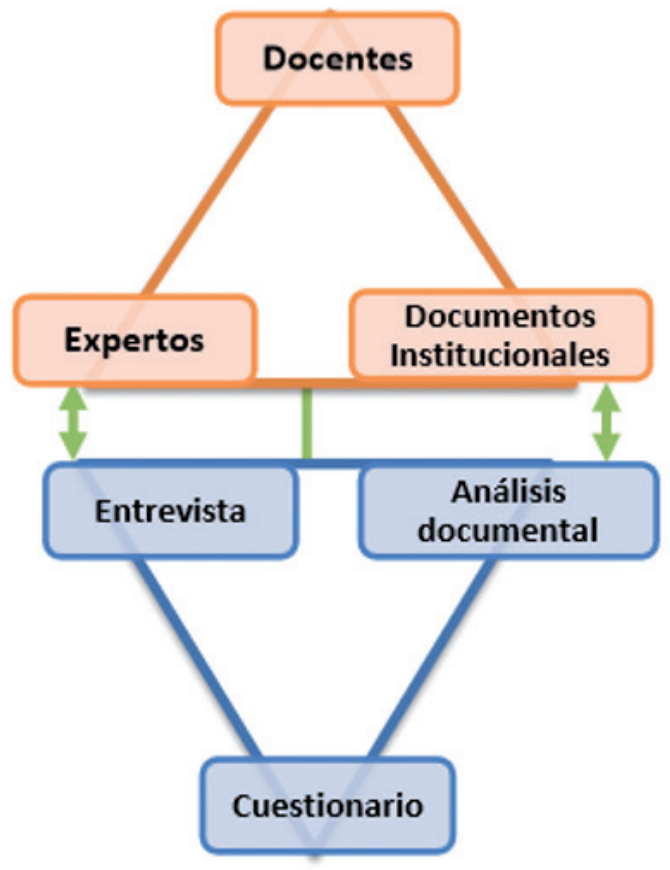

Figura 2. Triangulación de fuentes y técnicas. Elaboración propia. 
Para la recogida de datos se diseña y aplica de manera online un cuestionario validado en estudio preliminar. Se realiza también un diseño de entrevista semiestructurada y de Focus Group aplicado a grupos de expertos de manera presencial (visitando las difernentes localidades en las que desempeñan su labor profesional). Finalmente se diseña un registro de categorías para la extracción de datos de los planes y programas de atención al alumnado extranjero de los centros de la muestra. En el apartado de instrumentos describiremos con más detalle el procedimiento de validación de los mismos.

La inteción de este triple análisis es conocer de qué manera se está respondiendo a la diversidad cultural en los centros educativos, qué tipo de respuestas educativas se están ofreciendo, desde qué enfoque se está trabajando y cuál es la implicación de los profesionales del centro -no sólo de los que desarrollan o apoyan directamente la educación del alumnado de origen extranjero- sino de todo el claustro y, por extensión, de toda la comunidad educativa.

Para la realización de dichos análisis y dependiendo de la naturaleza de los datos y del diseño metodológico propuesto, se han utilizado principalmente dos softwares especializados, el programa SPSS para el análisis estadístico y validación del cuestionario y el programa NVivo para el análisis de los datos cualitativos procedentes de las entrevistas y Focus Groups.

Finalmente, nos referimos a la muestra del estudio:

A) En el caso de este diseño cuantitativo se ha utilizado un muestreo aleatorio simple (probabilístico). Se ha contado con 137 docentes de Infantil, Primaria y Secundaria que han respondido el cuestionario validado al efecto. Se trata de profesorado de centros educativos de 56 localidades de toda la región extremeña, con independencia de la existencia o no de programas específicos de atención al alumnado extranjero o similares implementados en su centro. De los137 docentes encuestados, 80 son mujeres y 47 son hombres y más de la mitad se encuadran en la franja de edad de 36 a 45 años, siendo el siguiente grupo más numeroso el del casi $25 \%$ que se incluye en el grupo de 26 a 35 años. En cuanto al perfil del grupo, el 23,91\% desempeñan su labor docente en la etapa de Infantil, el 48,55\% en la de Primaria, el 34,06\% en la de Secundaria Obligatoria y el $25,36 \%$ restante en Bachillerato. La mayor parte de ellos afirman tener experiencia en el trabajo con alumnado 
La Interculturalidad en Extremadura: tareas pendientes para una escuela inclusiva del s. XXI

Raquel Borrero López y Florentino BlázQuez Entonado

extranjero (111 docentes) y con alumnado de minorías étnicas o culturales (109 docentes), en muchos de los casos coincidentes ambas experiencias. Ciertamente, la gran mayoría de la muestra tiene o ha tenido experiencia con la diversidad cultural, interés que también ha podido motivar de algún modo su participación en el presente estudio. De los centros de los 137 encuestados, casi el $90 \%$ pertenece a centros de titularidad pública, el resto de la muestra lo hace en centro concertado a excepción de un sujeto que desempeña su función en centro privado, porcentajes muy ajustados a la distribución de centros en la comunidad autónoma.

B) Mediante la aplicación de un muestreo deliberado (no probabilístico) se cuenta con 40 expertos del ámbito de la educación. Por un lado se delimita un grupo de 34 asesores, orientadores, profesionales experimentados en el trabajo con alumnado de origen extranjero de diferentes localidades de la región extremeña, con quienes se realizan 1 entrevista y 9 grupos de discusión (focus group); por otro lado, se delimita un grupo de investigadores y docentes de educación superior, de ámbito nacional e internacional, dedicados a la formación del profesorado, grupo al que pertenece los 6 sujetos restantes y con quienes se realizan 1 entrevista y 2 grupos de discusión (focus group).

C) Y en tercer lugar, para el análisis documental se utilizan los documentos institucionales de 22 centros educativos acogidos al Programa de Atención al Alumnado Inmigrante o centros educativos con elevado número de alumnado extranjero o de minorías étnicas y culturales de Extremadura. En la selección de la muestra de este estudio vuelve a utilizarse un muestreo deliberado (no probabilístico) que además podríamos definir, por las circunstancias vinculadas al proceso de elección y diseño, como muestra de oportunidad al haber utilizado como fuente de la documentación objeto de análisis, parte de los centros implicados en las muestras anteriores. Así, hemos contado con los $\mathbf{1 7}$ centros del Programa de Atención al Alumnado Inmigrante y con los $\mathbf{5}$ centros en los que hemos llevado a cabo las entrevistas y Focus group en las provincias de Cáceres y Badajoz, en los que se escolariza un elevado número de alumnado extranjero $y / o$ perteneciente a grupos minoritarios. $Y$ son procedentes de Badajoz (7), Almendralejo (6), y uno respectivamente de Don Benito, Olivenza y Villanueva de la Serena (todos 
de la provincia de Badajoz) más 3 de Talayuela y 2 de Navalmoral de la Mata (provincia de Cáceres). Con la autorización correspondiente, los equipos directivos de los centros han aportado los datos referidos al alumnado de origen extranjero escolarizado, así como los Planes de Acogida y Atención al Alumnado Inmigrante, documentación institucional de los centros recogida como objeto de análisis.

Tabla 1. Descripción de técnicas y participantes. Elaboración propia.

\begin{tabular}{|c|c|c|c|}
\hline Técnica & Participantes & Cualidad & Análisis \\
\hline Cuestionario & $\begin{array}{l}137 \text { docentes de } 56 \text { lo- } \\
\text { calidades extremeñas }\end{array}$ & $\begin{array}{l}\text { Trabajan con alumnado de } \\
\text { origen extranjero de Infan- } \\
\text { til, Primaria y Secundaria }\end{array}$ & $\begin{array}{l}\text { Estadístico } \\
\text { Descriptivo } \\
\text { SurveyMonkey } \\
+ \text { SPSS }\end{array}$ \\
\hline $\begin{array}{l}\text { Entrevistas se- } \\
\text { miestructuradas } \\
\text { y Focus Group. }\end{array}$ & $\begin{array}{l}34 \text { docentes experi- } \\
\text { mentados en trabajo } \\
\text { con inmigrantes. }\end{array}$ & $\begin{array}{l}\text { Asesores/as, orientadores/ } \\
\text { as... }\end{array}$ & $\begin{array}{l}\text { Cualitativo } \\
\text { QSR NVivo }\end{array}$ \\
\hline Grupo Experto & $\begin{array}{l}6 \text { expertos investigado- } \\
\text { res universitarios }\end{array}$ & $\begin{array}{l}\text { Centros de Enseñanza de } \\
\text { Educación Superior }\end{array}$ & \\
\hline $\begin{array}{l}\text { Análisis Do- } \\
\text { cumental de } \\
\text { Contenido } \\
\end{array}$ & 24 centros educativos & $\begin{array}{l}\text { Acogidos al Programa de } \\
\text { Atención al Alumnado } \\
\text { Inmigrante o similar }\end{array}$ & $\begin{array}{l}\text { Cualitativo } \\
\text { Registro cate- } \\
\text { gorizado } \\
\end{array}$ \\
\hline
\end{tabular}

\subsection{Instrumentos de acceso a los datos}

Para el diseño y desarrollo de todos y cada uno de los instrumentos se han tenido en cuenta los criterios de control pertinentes. Describimos a continuación algunas características relevantes del diseño de los mismos:

a) El cuestionario. Para determinar la validez de contenido, inicialmente se somete a juicio de seis expertos y utilizando la fórmula de Lawshe (1975) en la actualización propuesta por Tristán (2008), quien establece un valor mínimo de razón de validez de 0,62 para asegurar que los criterios de unanimidad no se deban al azar. Tras las aportaciones de todos los jueces, el cuestionario obtuvo un IVC (Índice de Validez de Contenido) de 0,82 y fueron eliminados los ítems que presentaron un valor muy inferior al mínimo de razón de validez establecido por Lawshe. En segundo lugar, se realizó la validez de constructo llevada a cabo con mediante la técnica de análisis factorial. Para estimar fiabilidad del 
La Interculturalidad en Extremadura: tareas pendientes para una escuela inclusiva del s. XXI

Raquel Borrero López y Florentino BlázQuez Entonado

instrumento, es decir, el grado en que su aplicación repetida a un mismo sujeto producirá resultados iguales, se empleó el Alfa de Cronbach. El resultado de la prueba indica una fiabilidad de $=0,874$ tratándose de un coeficiente adecuado para determinar la confiabilidad de la prueba. Las categorías en torno a las cuales se aglutinan los ítems de contenido tras la aplicación de las diferentes pruebas de validez y fiabilidad son las cinco siguientes: (1) Apoyo educativo; (2) El profesorado (desempeño profesional y formación); (3) El trabajo de área y aula; y (4) La convivencia escolar: Comunidad Educativa y participación. La versión final del instrumento queda recogida en el trabajo de Borrero (2016).

b) La entrevista se concreta en su aplicación mediante dos procedimientos, la técnica de entrevista individual propiamente dicha y la técnica de Focus Group o grupos de discusión en torno a un problema definido. El instrumento se diseña para llevar a cabo un proceso de entrevista-discusión de entre 45 y 60 minutos como máximo, objetivo logrado en la totalidad de los casos, con algo más de dilatación en los Focus Group más numerosos. Para poder garantizar la credibilidad y consistencia de la investigación cualitativa, el diseño de la entrevista también ha sido sometido a juicio experto. Además, se contempla el requisito de aplicar un mínimo de dos Focus Group para poder establecer análisis y comparaciones, sobradamente sobrepasado teniendo en cuenta las muestras sobre la que se ha aplicado el instrumento.

C) El registro de análisis documental establece un total de 21 categorías de análisis, sometidas igualmente a juicio de 6 expertos, entendidas como indicadores de la presencia de medidas de atención a la diversidad cultural en los centros educativos. El diseño parte de una adaptación del Cuestionario para la evaluación del tratamiento de los procesos e interculturalidd en los centros educativos de García Argüello (2003), profesor colaborador del Proyecto Atlántida para generar espacios escolares interculturales. Este registro categorizado nos ha permitido el análisis documental de PEC, PCC, Programas de Mejora, Proyectos Interculturales, Programaciones anuales o trimestrales, Adaptaciones Curriculares, Refuerzos Educativos y Programas de Educación Compensatoria para alumnos de origen extranjero o procedente de minorías culturales o étnicas facilitados por los centros educativos bajo la demanda de los planes de acogida y atención al alumnado de origen extranjero o minoritario. La presencia de los indicadores formulados de manera observable en el proyecto de centro (salvando la heterogeneidad de formatos ofrecidos 
desde los diferentes centros educativos) nos permitiría acercarnos a un modelo de centro educativo intercultural respetuoso con el paradigma de la diversidad cultural que defendemos. Dadas las limitaciones de espacio, no se reflejan aquí las categorías de análisis, pero también pueden consultarse en el trabajo ya citado de Borrero (2016).

\section{Resultados}

Los datos que han sido analizados e interpretado desde el paradigma cuantitativo (los resultantes de la aplicación del cuestionario) se ordenan en torno a las cinco categorías confirmadas mediante el análisis factorial aplicado.

Recordemos que en esta parte del trabajo contamos con las respuestas de 83 mujeres y 54 hombres, profesorado en activo en centros educativos de Infantil, Primaria y Secundaria de titularidad pública, concertada y privada. Para el desarrollo de dicho análisis se ha aplicado la técnica de análisis estadístico descriptivo mediante la realización de análisis de frecuencias a partir de los softwares SPSS y SurveyMonkey.

Las respuestas de los encuestados se reflejan en torno a las siguientes categorías finalmente obtenidas: Apoyo educativo; El profesorado (desempeño profesional y formación); El trabajo de área y aula; y La convivencia escolar: Comunidad Educativa y participación. Para el desarrollo de la categorización se realiza un análisis factorial sobre una muestra amplia (137 sujetos) y representativa (procedentes de localidades de toda la región) capaz de garantizar la validez externa. Así, para determinar si era posible realizar un análisis factorial, se verificó la medida de adecuación muestral KMO (Kayser, Meyer y Olkin) y la prueba de Bartlett. Ambos fueron adecuados al arrojar coeficientes de 0,922 y 0 respectivamente.

Optamos por la extracción de factores mediante el análisis de los componentes principales y rotación Varimax, lo cual arrojó 5 factores principales que explican el $49,85 \%$ acumulado de la varianza total, lo que nos permite mantener la distribución inicial de las variables en 5 categorías. Los resultados se encuentran en la siguiente tabla: 
La Interculturalidad en Extremadura: tareas pendientes para una escuela inclusiva del s. XXI

Raquel Borrero López y Florentino BlázQuez Entonado

Tabla 2. Porcentaje de varianza total explicada. Elaboración propia.

\begin{tabular}{|c|c|c|c|c|c|c|}
\hline \multirow{2}{*}{ Componente } & \multicolumn{3}{|c|}{ Autovalores iniciales } & \multicolumn{3}{|c|}{$\begin{array}{l}\text { Sumas de extracción de cargas } \\
\text { al cuadrado }\end{array}$} \\
\hline & Total & $\begin{array}{c}\text { \% de } \\
\text { varianza }\end{array}$ & $\begin{array}{c}\% \% \\
\text { acumulado } \\
\end{array}$ & Total & $\begin{array}{c}\text { \% de } \\
\text { varianza }\end{array}$ & $\begin{array}{c}\% \% \\
\text { acumulado } \\
\end{array}$ \\
\hline 1 & 25,745 & 22,387 & 22,387 & 25,745 & 22,387 & 22,387 \\
\hline 2 & 13,251 & 11,522 & 33,909 & 13,251 & 11,522 & 33,909 \\
\hline 3 & 7,186 & 6,248 & 40,157 & 7,186 & 6,248 & 40,157 \\
\hline 4 & 6,071 & 5,279 & 45,436 & 6,071 & 5,279 & 45,436 \\
\hline 5 & 5,082 & 4,419 & 49,855 & 5,082 & 4,419 & 49,855 \\
\hline 6 & 4,689 & 4,078 & 53,933 & 4,689 & 4,078 & 53,933 \\
\hline 7 & 4,449 & 3,869 & 57,802 & 4,449 & 3,869 & 57,802 \\
\hline 8 & 3,773 & 3,281 & 61,083 & 3,773 & 3,281 & 61,083 \\
\hline 9 & 3,580 & 3,113 & 64,196 & 3,580 & 3,113 & 64,196 \\
\hline 10 & 3,462 & 3,011 & 67,207 & 3,462 & 3,011 & 67,207 \\
\hline 11 & 3,077 & 2,676 & 69,882 & 3,077 & 2,676 & 69,882 \\
\hline 12 & 2,988 & 2,599 & 72,481 & 2,988 & 2,599 & 72,481 \\
\hline 13 & 2,614 & 2,273 & 74,755 & 2,614 & 2,273 & 74,755 \\
\hline 14 & 2,400 & 2,087 & 76,841 & 2,400 & 2,087 & 76,841 \\
\hline 15 & 2,202 & 1,914 & 78,756 & 2,202 & 1,914 & 78,756 \\
\hline 16 & 2,099 & 1,826 & 80,581 & 2,099 & 1,826 & 80,581 \\
\hline 17 & 1,794 & 1,560 & 82,141 & 1,794 & 1,560 & 82,141 \\
\hline 18 & 1,731 & 1,505 & 83,647 & 1,731 & 1,505 & 83,647 \\
\hline 19 & 1,619 & 1,408 & 85,055 & 1,619 & 1,408 & 85,055 \\
\hline 20 & 1,551 & 1,349 & 86,404 & 1,551 & 1,349 & 86,404 \\
\hline 21 & 1,472 & 1,280 & 87,684 & 1,472 & 1,280 & 87,684 \\
\hline 22 & 1,403 & 1,220 & 88,904 & 1,403 & 1,220 & 88,904 \\
\hline 23 & 1,225 & 1,065 & 89,969 & 1,225 & 1,065 & 89,969 \\
\hline 24 & 1,155 & 1,004 & 90,973 & 1,155 & 1,004 & 90,973 \\
\hline 25 & 1,110 &, 965 & 91,938 & 1,110 &, 965 & 91,938 \\
\hline 26 & 1,008 & 877 & 92,815 & 1,008 & 877 & 92,815 \\
\hline
\end{tabular}

Un análisis aproximativo de la matriz de componentes rotados permitió establecer que si bien la cantidad de dimensiones se ajustaba a la propuesta originalmente (5), los ítems que las conformaban no concordaban con los resultados del análisis factorial, pues algunos reactivos aparecían en dimensiones que no les correspondían. Del mismo modo, el análisis de componentes principales nos permitió suprimir algunas 
variables prescindibles por su menor correlación, concretamente 26 de ellas, lo que a su vez nos ayuda a compensar el punto débil del excesivo tiempo invertido (superior al deseable). Para superar esta situación, se procedió a analizar estos ítems en función de su saturación en otras dimensiones y el grado de adecuación que presentaban en los nuevos factores $y$, posteriormente, se realizaron los cambios respectivos. A efectos de conocer si estas modificaciones afectarían de manera negativa la validez de constructo, se procedió a realizar un análisis factorial de segundo orden con las modificaciones generadas a partir del análisis de primer $\mathrm{KMO}$ y prueba de Bartlett. La versión final del mismo obtenida tras este trabajo, se analiza estadísticamente para generar un instrumento mejorado, fiable y válido. El espacio con el que contamos para la exposición de este trabajo nos impide reflejar los gráficos de frecuencias de cada uno de los ítems del cuestionario (87 en la versión final del instrumento) y su análisis pormenorizado, motivo por el cual incluimos la siguiente síntesis: Una síntesis de los resultados derivados del análisis de los datos en torno a las diferentes categorías tenidas en cuenta, se presenta a continuación:

Con respecto a la categoría que hemos denominado Apoyo Educativo, las opiniones del profesorado y las prácticas desarrolladas en los centros educativos en los que desempeñan su tarea, se corresponden mayoritariamente - al menos conceptualmente- con muchos de los principios básicos que han de ser tenidos en cuenta, según nuestra propuesta, a la hora de ofrecer una adecuada respuesta educativa al alumnado en contextos caracterizados por la diversidad cultural. Los profesionales encuestados suelen mostrar acuerdo con la mayoría de ítems que tienen en cuenta la atención a la diversidad, siendo también conscientes de la influencia que las características vinculadas a las diferencias culturales imprimen al proceso educativo del alumnado. Los datos cualitativos permiten observar una cierta disonancia en la idea de educación intercultural que entienden y desean y aquella que practican. El profesorado se siente interpelado por las necesidades educativas que se presentan en los contextos multiculturales en los que desempeñan su labor; sin embargo, no siempre la rigidez del sistema o la autonomía que a la hora de contextualizar las estrategias educativas con capaces de implementar en sus centros, llegan a mostrar una total equivalencia entre el discurso y la práctica.

En cuanto la categoría sobre el Desempeño y formación del profeso- 
La Interculturalidad en Extremadura: tareas pendientes para una escuela inclusiva del s. XXI

Raquel Borrero López y Florentino BlázQuez Entonado

rado, los resultados arrojan una actitud muy favorable hacia la asunción de la educación intercultural como modelo de enseñanza, aunque igualmente se observa la ausencia de estrategias, formación y apoyo suficientes para ponerla en marcha. Pero se detecta que el profesorado no puede caminar solo; son las universidades, las administraciones educativas, las instituciones de formación permanente y las entidades locales de desarrollo social, las instancias que deben respaldar su labor garantizando la calidad de la oferta formativa y la modificación de las estructuras. La perpetuación de los modelos vigentes por parte del sistema y la lentitud de los cambios que poco a poco se generan y la asistematicidad de la dotación de recursos dependiendo de la época de carestía o no por parte de la Administración, generan actitudes de pasividad, ausencia de implicación y desencanto del profesorado.

Con respecto a la categoría de Trabajo de área y aula, el hecho multicultural se ha hecho presente en los centros educativos extremeños y en ellos, a su vez, la transformación en escuelas interculturales cuando empiezan a ofrecer respuestas a la diversidad cultural. Si bien no cualquier tipo de respuesta, ya que al reconocer esta realidad como elemento presente en el contexto y tratar de incorporarla como componente de sus proyectos institucionales, ésta adquiere un carácter propio y ajustado, aunque en este desarrollo, no todos los intentos son exitosos y no siempre el profesorado puede librarse de la frustración que siente al no encontrar la implicación deseada en el alumnado y las familias. La incorporación de la competencia intercultural en las diferentes áreas curriculares y el trabajo de todas sus dimensiones es, por el momento, una tarea pendiente. Sin embargo, el profesorado parece haber tomado consciencia de la necesidad de adaptar sus programaciones a la diversidad cultural con la que trabaja y teniendo en cuenta todo tipo de estrategias metodológicas, de convivencia y de resolución de conflictos capaces de generar el clima más apropiado.

En cuanto a la categoría sobre Convivencia escolar, observamos que son muchos los aspectos a tener en cuenta para garantizar la verdadera inclusión del alumnado de diferentes orígenes, etnias y culturas; y para desarrollar estrategias útiles de resolución de conflictos garantes de un clima escolar apropiado para la convivencia y el aprendizaje. Los profesores no asimilan la diversidad cultural a un mayor grado de violencia o a la aparición de mayor número de conflictos (aunque durante las entrevistas se ha observado en bastantes casos esta asociación). A tal efecto el 
profesorado considera como una necesidad la de trabajar conjuntamente para establecer planes de convivencia, planificar medidas y estrategias específicas de resolución de conflictos y diseñar y desarrollar planes de acción tutorial que contribuyan al desarrollo integral del alumnado.

En torno a la última categoría, Comunidad educativa y participación, los datos indican que se asume que una educación intercultural y el desarrollo de la competencia intercultural han de incluir la participación de la familia y del entorno, contando con ellos para el desarrollo de prácticas y aprendizajes integrales. Por lo general, el profesorado está de acuerdo con estos principios aunque no se cuentan con estrategias efectivas para la implicación de las familias de alumnado extranjero en los centros, al igual que tampoco parece existir una coordinación fluida entre los diferentes agentes sociocomunitarios.

Por otro lado se presentan los datos que han sido analizados e interpretados desde el paradigma cualitativo. Recordemos que en esta parte del trabajo hemos contado con el discurso de 40 sujetos, entrevistados mediante la combinación de la metodología tradicional de entrevista individual y, en la mayor parte de casos, mediante la técnica de entrevista grupal del Focus Group (grupos de discusión focalizados) distribuidos en torno a 12 casos o momentos de entrevista diferenciados. Los resultados se ordenan en torno a las tres categorías o bloques de contenido de la propia entrevista, centrada en la explicación de la competencia intercultural del profesorado. Para el desarrollo del análisis de contenido se ha aplicado el método inductivo a partir del sistema de categorías diseñadas desde la teoría que actúan como hipótesis de cara a la interpretación de la información resultante.

Para la realización de los análisis se ha contado con la versión 9 del paquete de análisis cualitativo NVivo.En torno a las categorías diseñadas y a su orientación se han ido generando una serie de patrones y temas que han ido dando cabida a los datos reducidos de los textos transcritos. Las categorías (Cat.) y subcategorías (Subcat.) de análisis aparecen reflejadas en la primera de las tablas que a continuación incluimos; la segunda recoge, a modo de informe del proceso de investigación, la referencia de la frecuencia total (Ref.) de codificación por caso, es decir, por entrevista - focus group (Caso 1, Caso 2, Caso 3, etc.); la tercera tabla refleja la codificación por nodo, es decir, por categoría y subcategoría de codificación. 
La Interculturalidad en Extremadura: tareas pendientes para una escuela inclusiva del s. XXI

Raquel Borrero López y Florentino BlázQuez Entonado

Tabla 3. Categorización Estudio Cualitativo.

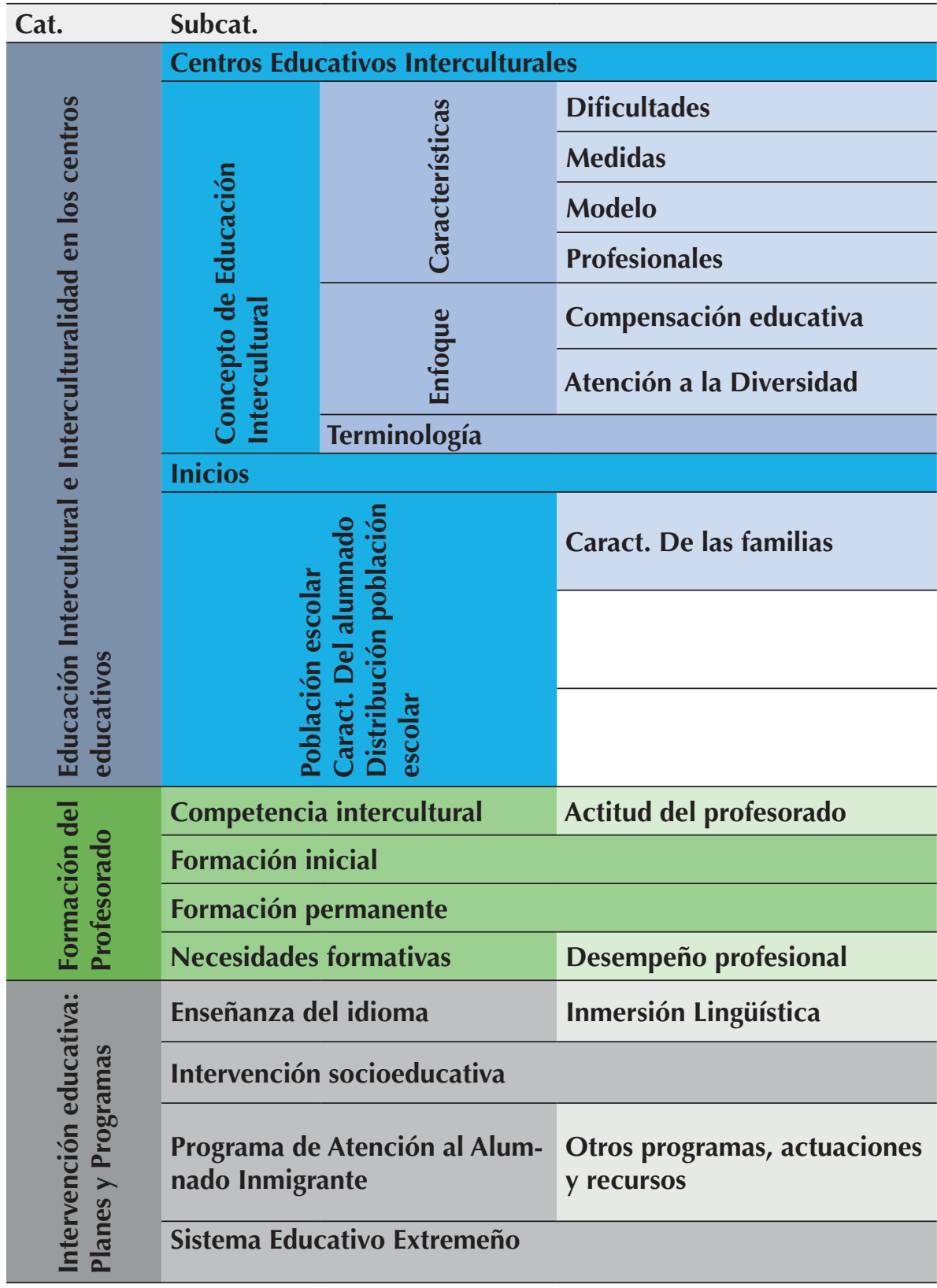


Tabla 4. Codificación de Casos Estudio Cualitativo.

\begin{tabular}{rccc} 
CASOS & $\begin{array}{c}\text { Referencias de } \\
\text { codificación }\end{array}$ & Palabras codificadas & Párrafos codificados \\
\hline Caso 1 & 30 & 10689 & 349 \\
\hline Caso 2 & 3 & 18421 & 730 \\
\hline Caso 3 & 30 & 9121 & 343 \\
\hline Caso 4 & 3 & 15822 & 694 \\
\hline Caso 5 & 6 & 18533 & 655 \\
\hline Caso 6 & 4 & 24747 & 924 \\
\hline Caso 7 & 33 & 11473 & 346 \\
\hline Caso 8 & 1 & 1986 & 118 \\
\hline Caso 9 & 25 & 11720 & 339 \\
\hline Caso 10 & 3 & 11739 & 597 \\
\hline Caso 11 & 2 & 8964 & 316 \\
\hline Caso 12 & 2 & 8964 & $\mathbf{5 7 2 7}$ \\
\hline Total & $\mathbf{1 4 2}$ & $\mathbf{1 5 2 1 7 9}$ & \\
\hline
\end{tabular}

Analizar la saturación de la codificación nos ha permitido identificar los principales núcleos de información. Por otra parte, la codificación se ha complementado con la vinculación entre las categorías que acumulan mayor porcentaje de codificación, realizando el análisis de la interacción entre las mismas. Se han establecido 5 resultados en función de las interacciones entre dichas categorías principales, que nos han permitido seleccionar las aportaciones más relevantes de esta parte del estudio, esclareciendo la toma de decisiones a la hora de priorizar los fragmentos de texto más representativos.

Tabla 5. Relación entre categoría.

\begin{tabular}{|lccc}
\hline RESULTADOS DE RELACIÓN & $\begin{array}{c}\text { Referencias de } \\
\text { codificación }\end{array}$ & $\begin{array}{c}\text { Palabras } \\
\text { codificadas }\end{array}$ & $\begin{array}{c}\text { Párrafos } \\
\text { codificados }\end{array}$ \\
\hline $\begin{array}{l}\text { Results } \backslash \backslash \text { Concepto de Educa- } \\
\text { ción Intercultural y Necesidades } \\
\text { Formativas }\end{array}$ & 99 & 15652 & 416 \\
\hline $\begin{array}{l}\text { Results } \backslash \backslash \text { Concepto Educación } \\
\text { Intercultural y Competencia } \\
\text { Intercultural }\end{array}$ & 99 & 15652 & 416 \\
\hline $\begin{array}{l}\text { Results } \backslash \backslash P \text { Planes y Programas y } \\
\text { Formación del Profesorado }\end{array}$ & 99 & 15652 & 416 \\
\hline
\end{tabular}


La Interculturalidad en Extremadura: tareas pendientes para una escuela inclusiva del s. XXI

Raquel Borrero López y Florentino BlázQuez Entonado

\begin{tabular}{lccc}
\hline RESULTADOS DE RELACIÓN & $\begin{array}{c}\text { Referencias de } \\
\text { codificación }\end{array}$ & $\begin{array}{c}\text { Palabras } \\
\text { codificadas }\end{array}$ & $\begin{array}{c}\text { Párrafos } \\
\text { codificados }\end{array}$ \\
\hline $\begin{array}{l}\text { Results } \backslash \text { Relación Entre Educa- } \\
\text { ción Intercultural y Formación } \\
\text { del Profesorado }\end{array}$ & 99 & 15652 & 416 \\
\hline $\begin{array}{l}\text { Results } \backslash \text { Relación entre Planes } \\
\text { y Programas y Educación Inter- } \\
\text { cultural }\end{array}$ & 99 & 15652 & 416 \\
\hline
\end{tabular}

Finalmente, para el análisis de los modelos de educación intercultural de los centros educativos extremeños a partir de sus programas institucionales se realiza una exposición descriptiva del contenido relevante de los documentos de los centros y a continuación se aplica la categorización diseñada a partir de revisión bibliográfica y validación experta, como ya recogimos en el apartado de descripción de los instrumentos (3.1).

Se aprecia en los datos una tendencia a asimilar educación intercultural con inmigración y, por consiguiente, con multiculturalidad. La sensibilización y la formación son imprescindibles en este sentido ya que la inmigración, tal y como se entiende en la actualidad y, concretamente en Extremadura, ha adquirido unas ciertas connotaciones que sesgarían la descripción de la diversidad cultural en sí, ya que en nuestros centros educativos se han vinculado especialmente con la acogida de inmigrantes y mayoritariamente en situación de pobreza. La población inmigrante que llega a nuestra Comunidad Autónoma posee unas características muy concretas, transferidas desde su estrato sociocultural y económico.

La región estudiada es hoy una región caracterizada por la diversidad cultural, ya no se podría continuar hablando de un fenómeno, sino de una realidad. Y son las realidades y los hechos los que desencadenan respuestas y transformaciones, en este caso, la interculturalidad que estudiamos. Sin embargo, nos encontramos a mitad del camino, profundizando progresivamente en una serie de conceptos que aún no se han traducido en actitudes y acciones coherentes.

Las opiniones del profesorado se muestran de acuerdo en que las actividades interculturales tienen que ir dirigidas a toda la población escolar, sin exclusión. Pero todavía son escasos los docentes que contemplan la interculturalidad como una propuesta crítica y transformadora de la educación. 


\section{Conclusiones}

A través de las opiniones del profesorado y de los expertos hemos extraído una serie de consideraciones en torno al conocimiento sobre la educación intercultural, a los modelos de intervención y de formación existentes, a la participación y, finalmente, a la convivencia y al clima escolar. Se ha delimitado y comprendido, a través de la revisión de la normativa vigente y reguladora de las actuaciones con el alumnado perteneciente a minorías étnicas y/o de origen extranjero, el modelo que nuestro sistema educativo extremeño y concretamente la administración educativa competente, está promoviendo para el desarrollo de una educación intercultural. Basando su funcionamiento en el principio de la compensación educativa y en una intervención por programas, llegamos a la conclusión de que en los centros extremeños no hablamos todavía de centros interculturales, sino de centros en los que existe diversidad cultural y cuya respuesta a la misma es de carácter normativo y multicultural. Superada está, al menos en el plano cognitivo que se desprende de los datos y del discurso de los profesionales, la barrera del rechazo, aunque sus manifestaciones resultan cíclicas y se ven condicionadas por el tamaño de la población de acogida y las actitudes y predisposición de la misma a la asimilación; sin embargo y de manera general, no se han desarrollado las medidas organizativas y didácticas más coherentes con modelos educativos interculturales o basados en el diálogo intercultural

Para el desarrollo de una verdadera educación intercultural, sería determinante que el profesorado concibiese la interculturalidad no como una modalidad especial de educación para el alumnado inmigrante, sino como una propuesta de acción educativa que ha de darse, como hemos dicho, no solamente en los centros educativos en los que hay alumnado inmigrante, sino en todos, en general.

Lo más destacado de los resultados analizados es que la respuesta educativa que hay que ofrecer en contextos de diversidad cultural es interpretada como una modalidad diferente de atención educativa. Sin embargo, trabajar en esta línea no permite hablar de escuela inclusiva, ni de un currículo compartido, mostrándose en las prácticas de intervención esa dicotomía existente aún con respecto a la mejor manera de trabajar con las diferencias más que como valor añadido, como problema que se debe solucionar o carencia que se debe compensar.

Como conclusión general de este trabajo se desprende que el mo- 
La Interculturalidad en Extremadura: tareas pendientes para una escuela inclusiva del s. XXI

Raquel Borrero López y Florentino BlázQuez Entonado

delo educativo subyacente a la realidad multicultural oscila entre un modelo de naturaleza asimilacionista y un modelo compensatorio, dentro de las tres orientaciones que recoge la bibliografía: asimilacionista, segregador-compensador y enfoque crítico intercultural existiendo por lo tanto, una escasa repercusión en la práctica de la percepción que tiene el profesorado sobre las implicaciones de la multiculturalidad en el ámbito educativo, especialmente en el trabajo cotidiano en el aula y los modelos de comunicación y disciplinares que en ella se emplean.

Ahora bien, aun cuando el profesorado se encuentra todavía lejos de haber interiorizado un modelo educativo intercultural, se aprecian preocupación e intereses auténticos por este alumnado de grupos minoritarios, lo que constituirá un firme punto de partida para el trabajo formativo. El problema puede venir de la identificación plena de los proyectos de educación intercultural con los proyectos de educación compensatoria, lo que lógicamente nos llevaría a plantear otras discusiones acerca del carácter compensatorio de las acciones educativas interculturales. Ya hemos señalado como punto fuerte dentro del proceso de cambio, el hecho de que el profesorado haya decidido ir transformando progresivamente sus prácticas. La organización de actividades que recogen la diversidad cultural de los centros dirigidas a todo el alumnado y a toda la comunidad educativa, así lo indica. Sin embargo, el riesgo que esta tendencia conlleva es la identificación de la educación intercultural con celebraciones lúdico-festivas, que no sería más que un acercamiento superficial y folclórico desde el currículo.

Queremos cerrar este apartado de conclusiones reflejando las tareas pendientes a las que nos referíamos en el título de este trabajo para el desarrollo de una escuela inclusiva propia siglo XXI y las cuestiones que se deben tomar en consideración para marcar los horizontes próximos de la formación de profesionales de la docencia para una educación intercultural crítica. La construcción de un nuevo modelo basado en la promoción de escuelas interculturales pasaría por revisar algunos de los principales retos de la escuela inclusiva:

- La primera tarea sería pasar de forma inminente de una concepción de la educación intercultural desde su más arraigado origen compensatorio a un modelo de escuela nueva, participada e interactiva. Los estudiantes extranjeros o procedentes de minorías no necesitan, en muchos casos, aprender castellano o nuestra cultura, pero les hacemos ver que sin esos elementos no pueden aportar 
nada a la escuela, mucho menos a la sociedad. Hasta que no saben hablar nuestro idioma, hasta que no son un poco más parecidos al resto no pueden participar de las clases y en este sentido minamos su autoestima y condicionamos el desarrollo de su identidad. Les asimilamos hasta que pueden "integrarse" en el ritmo ordinario de un aula "normalizada". La educación intercultural no es educación de inmigrantes y la educación que se centra en la inmersión lingüística y la compensación, no es educación intercultural desde el modelo que defendemos. La educación intercultural también ha de evaluar la respuesta capaz de transformar las diferencias de las culturas que entran en contacto en oportunidades de aprendizaje, éxito y mejora como ya se ha señalado.

- La segunda tarea supondría generar normativa al servicio de las verdaderas necesidades y no al revés. Más que reproducir recetas o modelos que conducen al fracaso, debemos emprender éxitos avalados por el bienestar afectivo, social y académico de los estudiantes, por su participación real y efectiva en el ámbito escolar y social.

- La tercera tarea sería la unión y la configuración de redes de trabajo. Reforzar las prácticas escolares de éxito que permiten constituir la base de una verdadera red de centros educativos interculturales capaces de promover el crecimiento en red, compartiendo esfuerzos en lugar de duplicándolos.

- La cuarta tarea sería propiciar una organización escolar y un liderazgo diferentes a través de la creación de Comunidades de Aprendizaje, lo que a su vez constituye una de las recomendaciones de la Comisión Europea. Para ello es imprescindible contar con tiempos y espacios institucionalizados para trabajar en estos retos y para compartir experiencias y avances.

- La quinta tarea sería adecuar la formación del profesorado a modelos pedagógicos apropiados. Quizás, una de las evidencias más importantes de que no se ofrece la mejor respuesta para los docentes es la insatisfacción bastante generalizada que se ha detectado en este trabajo. La estructura básica de un sistema inclusivo reconoce como imprescindibles la satisfacción y la percepción de logro a través de la implicación y el trabajo en equipo para generar un proyecto de cambio común.

- La última tarea consistiría en contar con una administración 
La Interculturalidad en Extremadura: tareas pendientes para una escuela inclusiva del s. XXI

Raquel Borrero López y Florentino BlázQuez Entonado

comprometida e implicada, y equipos directivos colegiados o capaces de desarrollar un liderazgo compartido. Ingredientes estos capaces de dotar de una verdadera autonomía a los centros y al profesorado, así como garantizar -sin fiscalizar- la calidad y la equidad de la respuesta educativa. Los docentes necesitan tiempos y espacios para compartir, para hablar de sus preocupaciones y para plantear sus soluciones y una buena fórmula, sin duda, son los grupos de trabajo.

Junto a todo, la voluntad sigue siendo el elemento motivador fundamental para el duro trabajo docente que cualquier transformación, incluida la de la escuela extremeña requiere. No hay nada particularmente nuevo sobre esta realidad. Sin embargo, las formas de la distribución inequitativa siempre se están ajustando, a menudo en respuesta a los avances logrados por los movimientos creados para interrumpir la injusticia entre los que este modesto trabajo, como tantos del Dr. Escudero, intenta encontrarse.

\section{Referencias bibliográficas}

Aguado, M.T. (2003). Pedagogía intercultural. Madrid: McGraw-Hill.

Arnaiz; P. y de Haro R. (2004) Ciudadanía e interculturalidad: claves para la educación del Siglo XXI. Educatio s. XXI, 22. 19-37.

Banks J.A. y J. Lynch (Eds.) (1986). Multicultural Education in Western Societies. London: Holt, Rinehart and Winston.

Blázquez, F. (1999). Los proyectos institucionales, un espacio para la educación intercultural. En M. Lorenzo, M. y J.A. Ortega, Organización y Dirección de Instituciones Educativas Interculturales (pp. 74-94). Granada: Universidad de Granada.

Blázquez, F. y Borrero, R. (2012). Educación para todos y todas. La escuela Intercultural. En P. Gutiérrez, R. Yuste y R. Borrero (Coords.) La escuela inclusiva desde la innovación docente. Madrid: Libros de la Catarata.

Borrero, R. y Yuste, R. (2011). Digiculturalidad.com. Interculturalidad y TIC unidas en el desarrollo de un enfoque competencial del currículum. En J. Leiva y R. Borrero (Coords.), Interculturalidad y escuela. Perspectivas pedagógicas en la construcción comunitaria de la escuela intercultural (pp. 145-164). Barcelona: Octaedro.

Borrero, R. (2016). Estudio prospectivo para una formación intercultural del profesorado en la sociedad del siglo XXI. Tesis doctoral inédita. Universidad de Extremadura.

Castro, J. (2007). La inmigración en Extremadura. Análisis demográfico y distribución territorial. Revista de estudios extremeños, 63 (3), 1451-1520. 
La Interculturalidad en Extremadura: tareas pendientes para una escuela inclusiva del

Essomba, M.A. (2012). Inmigración, sociedad y educación en la UE. Hacia una política educativa de plena inclusión. Cultura y Educación, 24 (2), 137-148.

Esteve, J.M. (2004). La formación del profesorado para una educación intercultural. Bordón, 56 (1), 95-116.

García Argüello, J.M. (2003). Cuestionario para la Evaluación del tratamiento de los procesos de interculturalidad en centros educativos. En Proyecto Atlántida, Interculturalidad y Educación: Un nuevo reto para la sociedad democrática. Madrid: Proyecto Atlántida.

Gorski, P. (2009). What we're Teaching Teachers: An Analysis of Multicultural Teacher Education Coursework Syllabi. Teaching and Teacher Education, 5: 309-318.

Jiménez García, J.J. (2007). Diversidad de alumnos inmigrantes en Extremadura, una realidad más cercana. Revista de Estudios Extremeños, LXIII (3), 1521-1549.

Junta de Extremadura (2008). II Plan de Inclusión Social de Extremadura, 2008-2011. Badajoz: Consejería de Igualdad y Empleo.

Leiva, J.J. y Borrero, R. (Coords.) (2011). Interculturalidad y Escuela. Perspectivas Pedagógicas en la construcción comunitaria de la escuela intercultural. Barcelona: Octaedro.

León B; Gómez, T.; Felipe, E.; Gonzalo, M.; y Latas, C. (2008). Análisis psicoeducativo del alumnado inmigrante en Extremadura. Mérida: Junta de Extremadura.

Meer, N. y Modood, T. (2012): How does Interculturalism Contrast with Multiculturalism? Journal of Intercultural Studies, vol. 33, no 2, pp. 175-196.

Palaiologou; N. y Gorski, P. C. (2017) The evolution of intercultural and multicultural education: scholarship and practice for new sociopolitical and economic realities. Intercultural Education, vol. 28, 4, 353-355.

Ponce Solé, J. (2007). Segregación escolar e inmigración. Contra los guetos escolares: derecho y

políticas públicas urbanas. Centro de estudios políticos y constitucionales. Colección: Foro (16). Madrid: Inmigración y ciudadanía.

Tristán, A. (2008). Modificación al modelo de Lawshe para el dictamen cuantitativo de la validez de contenido de un instrumento objetivo. Avances en Medición, 6, 37-78. 
